\title{
A NEW SPECIES OF THE GENUS SQUALIUS BONAPARTE, 1837 (ACTINOPTERYGII, CYPRINIDAE) FROM THE TAGUS RIVER BASIN (CENTRAL SPAIN)
}

\author{
I. Doadrio ${ }^{1}$, S. Perea $^{1} \&$ F. Alonso ${ }^{2}$
}

\begin{abstract}
A new species of Squalius is described on the basis of morphological and genetic characters from the Gallo River and its tributaries (Tagus Basin) in central Spain. The new species is distinguished from other Iberian Squalius species by a combination of morphometric, meristic and genetic characters, such as wide infraorbital bones that do not cover the adductor mandibulae muscle; seven scales on the upper transversal line; small number of lateral line scales, mean $=39.8$ (39-42); a short and wide head, 4.3-4.9 $($ mean $=4.5)$ times the standard length; Short pectoral-ventral length, reaching 2.2-2.4 (mean $=2.3$ ) times the standard length. The new species differs from its closer taxa in terms of genetic GTR distances, $S$. carolitertii and $S$. pyrenaicus at 20 fixed nucleotide positions (molecular autapomorphies), in the mitochondrial cytochrome $b$ gene. Genetic divergence values for this new species and $S$. carolitertii and $S$. pyrenaicus are $3.8 \%$ and $6.1 \%$ respectively. The new species should be considered Critically Endangered (CR) according to the IUCN Red List Categories.
\end{abstract}

Key words: Cypriniformes, Cyprinidae, Taxonomy, Squalius castellanus, Gallo River, Tagus basin.

\section{RESUMEN}

Una nueva especie del género Squalius Bonaparte, 1837 (Actinopterygii, Cyprinidae) para la cuenca del Tajo (España Central)

Se describe una nueva especie de Squalius en base a caracteres morfológicos y genéticos. Esta nueva especie está restringida al río Gallo (cuenca del Tajo) en el centro de España y difiere del resto de las especies ibéricas de Squalius en una serie de caracteres morfométricos, merísticos y genéticos, tales como huesos infraorbitarios anchos pero que no cubren totalmente el músculo adductor mandibulae; siete escamas en la línea transversal superior, menor número de escamas en la línea lateral media = 39.8 (39-42); cabeza corta y ancha, siendo 4.3-4.9 (media = 4.5) veces la longitud estándar; longitud ventral-pectoral corta alcanzando 2.2-2.4 (media $=2.3)$ veces la longitud estándar. La nueva especie difiere de sus taxones más cercanos, en término de distancias geneticas GTR, en 20 posiciones nucleótidicas fijadas (autopomorfías moleculares) en el gen

Museo Nacional de Ciencias Naturales, CSIC, Department of Biodiversity and Evolutionary Biology, C/José Gutiérrez Abascal, 2, 28006 Madrid, Spain; mcnd147@mncn.csic.es; sperea@mncn.csic.es

2 Centro de Investigación Agraria de Albadalejito. Junta de Comunidades de Castilla-La Mancha. Cta. Toledo-Cuenca, Km 174. E-16194. Cuenca, Spain; falonso@jccm.es 
mitocondrial citocromo $b$. La divergencia genética entre la nueva especie descrita respecto a $S$. carolitertii y $S$. pyrenaicus para el gen citocromo $b$ fue de $3.8 \%$ y $6.1 \%$ respectivamente. Esta nueva especie debería ser considerada En Peligro Crítico (CR) de acuerdo con las Categorías de la Lista Roja de la IUCN.

Palabras clave: Cypriniformes, Cyprinidae, Taxonomía, Squalius castellanus, Río Gallo, cuenca del Tajo.

\section{Introduction}

The genus Squalius Bonaparte, 1837 is widely distributed throughout European freshwater lakes and rivers, and shows a high level of diversity in the Mediterranean area (Zardoya \& Doadrio, 1999; Durand et al., 2000; Doadrio \& Carmona, 2003). This genus includes approximately 15 species (Kottelat 1997; Doadrio \& Carmona 1998) distributed along the Mediterranean basin; two species are widely reported in Central and North Europe (Sanjur et al., 2003).

In the Iberian Peninsula, the genus Squalius is represented by eight endemic species that belong to the "Mediterranean lineage" (Sanjur et al., 2003): Squalius alburnoides (Steindachner, 1866), S. aradensis (Coelho, Bogutskaya, Rodrigues \& Collares-Pereira, 1998), $S$. carolitertii (Doadrio, 1987), $S$. malacitanus Doadrio \& Carmona, 2006, S. palaciosi (Doadrio, 1980), S. pyrenaicus (Günther, 1868), $S$. torgalensis (Coelho, Bogutskaya, Rodrigues \& Collares-Pereira, 1998) and S. valentinus Doadrio \& Carmona, 2006 and one species that belongs to the "Euro-Asiatic lineage", S. cephalus (Linnaeus, 1758), which is widely distributed throughout Europe (Sanjur et al., 2003). Squalius alburnoides and $S$. palaciosi are characterized as belonging to a different hybrid complex, and both species probably played an important role in the evolutionary history of Squalius (Carmona et al., 1997; Alves et al., 1997, 1999, 2001; Cunha et al., 2004).

Nevertheless, the taxonomy of Squalius in the Iberian Peninsula has been controversial because of inconsistencies between analyses based on genetic and morphological characters. Phenotypic plasticity is high among the species and diagnostic characters are difficult to find, but genetic approaches have demonstrated a high degree of differentiation between Iberian Squalius populations based on allozymes (Coelho et al., 1995; Alves et al., 1997; Carmona et al., 1997) and mitochondrial (mt) DNA sequences variation (Brito et al., 1997; Sanjur et al., 2003). These studies have focused mainly on populations of S. pyrenaicus and the high levels of genetic variation between these populations have resulted in the diagnoses and identification of four new species from the southern and eastern Iberian Peninsula, $S$. aradensis and $S$. torgalensis from Portugal (Coelho et al., 1998) and $S$ malacitanus and $S$. valentinus from Spain (Doadrio \& Carmona, 2006). Squalius carolitertii, which is also widely distributed throughout the north-western Iberian Peninsula, has not been as thoroughly studied, and no comparable levels of genetic variation have been found in initial studies of this species (Sanjur et al., 2003).

In this paper we reanalysed between-population genetic variation in Squalius carolitertii and $S$. pyrenaicus including a population from the upper Tagus Basin, which preliminary phenotypic examinations suggested as being distinct from both $S$. caroliterii and $S$. pyrenaicus. The purpose of this paper is to examine the phylogenetic relationships of this Tagus Basin population based on mitochondrial cytochrome $b$ sequence data, and to provide diagnostic morphological characters and a description of the new species of Squalius.

\section{Materials and methods}

\section{MORPHOMETRIC ANALYSES}

The description of this new Squalius species is based on the study of 21 individuals from the Gallo River and its tributaries (Tagus Basin). Only 17 adult specimens were used for comparative morphometric analysis. The holotype and a series of paratypes have been deposited in the Museo Nacional de Ciencias Naturales, Spain (MNCN). For comparative purposes we studied the following material: Squalius aradensis: MNCN243553-86, 34 ex. Algibre River, Querença, Portugal, leg I. Doadrio, 25/3/02. Squalius carolitertii: MNCN 26052, holotype, Cega River, Rebollo, Segovia prov., Duero Basin, leg. D. García de Jalón, 1/5/82, MNCN24141-24169, paratypes, 29 ex. Duero River, Peleagonzalo, Zamora prov., Duero Basin leg I. Doadrio and B. Elvira 14/8/81. Squalius malaci- 
Table 1.- Samples, collection locations, number of sampled populations in the tree and GenBank access numbers. GenBank Accession Numbers with * are new sequences obtained in this study.

Tabla 1.- Muestras, localidades, número de población en el árbol y número de acceso de GenBank. Los números de acceso de GenBank con* son secuencias nuevas obtenidas en este estudio.

\begin{tabular}{|c|c|c|c|c|}
\hline Species & Locality & River & $\begin{array}{c}\text { Basin (Population } \\
\text { designation) }\end{array}$ & $\begin{array}{c}\text { GenBank number } \\
\text { Accesion }\end{array}$ \\
\hline Squalius lucumonis & Istia d'Ombrone. Italy & Ombrone & Ombrone & AF421828 \\
\hline Squalius keadicus & Sparta. Grecia & Evrotas & Evrotas & AF090760 \\
\hline Squalius aradensis & Portugal & Arade & Arade 1 & AF421824 \\
\hline S. aradensis & Portugal & Arade & Arade 2 & AF421825 \\
\hline Squalius castellanus & Ventosa. Spain & Gallo & Tajo & DQ521423* \\
\hline Squalius carolitertii & Monzón de Campos. Spain & Pisuerga & Duero $(\mathrm{C} 1)$ & DQ521424* \\
\hline S. carolitertii & El Fresno. Spain & Adaja & Duero (C2) & AF421800 \\
\hline S. carolitertii & El Fresno. Spain & Adaja & Duero (C3) & AF045994 \\
\hline S. carolitertii & El Fresno. Spain & Adaja & Duero (C4) & AF421799 \\
\hline S. carolitertii & Bacones de Ojeda. Spain & Boedo & Duero (C5) & DQ521425* \\
\hline S. carolitertii & Bacones de Ojeda. Spain & Boedo & Duero (C6) & AF421797 \\
\hline S. carolitertii & Bacones de Ojeda. Spain & Boedo & Duero (C7) & AF421798 \\
\hline S. carolitertii & Peñarrubias de Pirón. Spain & Piron & Duero (C8) & DQ521427* \\
\hline S. carolitertii & Villanueva de Argaño. Spain & Hormazuelas & Duero (C9) & DQ521430* \\
\hline S. carolitertii & Santana. Spain & Valdivia & Duero (C10) & DQ521426* \\
\hline S. carolitertii & Riaza. Spain & Riaza & Duero (C11) & DQ521428* \\
\hline S. carolitertii & Cuéllar. Spain & Cega & Duero (C12) & DQ521429* \\
\hline S. carolitertii & Portugal & Vouga & Vouga (C13) & DQ521432* \\
\hline S. carolitertii & San Agustín. Spain & Bibei & Miño (C14) & AF421793 \\
\hline S. carolitertii & San Agustín. Spain & Bibei & Miño (C15) & AF421794 \\
\hline S. carolitertii & Rubia de Mixtos. Spain & Salas & Limia (C16) & AF421795 \\
\hline S. carolitertii & Rubia de Mixtos. Spain & Salas & Limia (C17) & AF421796 \\
\hline S. carolitertii & Portugal & Montego & Mondego (C18) & DQ521431* \\
\hline S. carolitertii & Portugal & Montego & Mondego (C19) & DQ521433* \\
\hline Squalius malacitanus & Guadaira. Málaga. Spain & Guadaira & (M1) & New sequence \\
\hline S. malacitanus & Benaojan. Málaga. Spain & Guadiaro & (M2) & New sequence \\
\hline Squalius pyrenaicus & Alcaraz. Spain & Guadalmina & Guadalquivir (P1) & AF421816 \\
\hline S. pyrenaicus & Alcaraz. Spain & Guadalmina & Guadalquivir (P2) & AF421817 \\
\hline S. pyrenaicus & Cañaveral de León. Spain & Montemayor & Guadalquivir (P3) & AF421790 \\
\hline S. pyrenaicus & Navas de Estena. Spain & Estena & Guadiana (P4) & AF421813 \\
\hline S. pyrenaicus & Casa de Fuente Vieja. Spain & Azuer & Guadiana (P5) & AF421804 \\
\hline S. pyrenaicus & Casa de Fuente Vieja. Spain & Azuer & Guadiana (P6) & AF421805 \\
\hline S. pyrenaicus & Ossa de Montiel. Spain & Lagunas de Ruidera & Guadiana (P7) & AF421822 \\
\hline S. pyrenaicus & Ossa de Montiel. Spain & Lagunas de Ruidera & Guadiana (P8) & AF421823 \\
\hline S. pyrenaicus & Uña. Spain & Laguna de Uña & Júcar (P9) & AF421806 \\
\hline S. pyrenaicus & Uña. Spain & Laguna de Uña & Júcar (P10) & AF421807 \\
\hline S. pyrenaicus & Álava. Spain & Río Baias & Ebro (P11) & DQ521434* \\
\hline S. pyrenaicus & Álava. Spain & Río Baias & Ebro (P12) & DQ521435* \\
\hline S. pyrenaicus & Álava. Spain & Río Baias & Ebro (P13) & DQ521436* \\
\hline S. pyrenaicus & La Adrada. Spain & Tiétar & Tajo (P14) & DQ521437* \\
\hline S. pyrenaicus & Jaraicejo. Spain & Almonte & Tajo (P15) & AF421791 \\
\hline S. pyrenaicus & Hoyos. Spain & Acebo & Tajo (P16) & AF421727 \\
\hline S. pyrenaicus & Cadalso de Gata. Spain & Arrago & Tajo (P17) & AF421726 \\
\hline S. pyrenaicus & Valverde del Fresno. Spain & Pesquero & Tajo (P18) & AF421811 \\
\hline S. pyrenaicus & Valverde del Fresno. Spain & Pesquero & Tajo (P19) & AF421712 \\
\hline S. pyrenaicus & Las Moedas. Spain & Bogarda & Segura (P20) & AF421820 \\
\hline S. pyrenaicus & Las Moedas. Spain & Bogarda & Segura (P21) & AF421821 \\
\hline Squalius valentinus & Sollana. Spain & Font de Barrent & Turia (V1) & AF421808 \\
\hline S. valentinus & Sollana. Spain & Font de Barrent & Turia (V2) & AF421809 \\
\hline S. valentinus & Callosa d'en Sarria. Spain & Algar & Algar (V3) & AF421818 \\
\hline S. valentinus & Callosa d'en Sarria. Spain & Algar & Algar (V4) & AF421819 \\
\hline S. valentinus & Beniarres. Spain & Serpis & Serpis (V5) & AF421810 \\
\hline S. valentinus & Beniarres. Spain & Serpis & Serpis (V6) & AF421815 \\
\hline Squalius torgalensis & Portugal & Torgal & Mira & DQ521438* \\
\hline
\end{tabular}


tanus: MNCN243699, holotype, Guadalmina River, Benahavis, Málaga prov., leg I. Doadrio, P. Garzón and R. Zardoya, 29/3/03, MNCN212280-311, paratypes, 32 ex., Guadalmina River, Benahavis, Málaga prov., Guadalmina Basin, leg. A. Doadrio, I. Doadrio jr., P. Garzón and I. Doadrio, 5/3/03. Squalius pyrenaicus: MNCN 24785, lectotype, Sintra, Portugal, leg. Günther MNCN189911-27, MNCN187359-70 29 ex., Montemayor River, Cañaveral de León, Huelva prov., Guadalquivir Basin, leg. I. Doadrio, 5/5/99. MNCN25716-18, 3 ex. Lozoya River River, Pontón de la Oliva, Madrid prov., Tajo Basin leg. I. Doadrio. MNCN69638-53, MNCN69685-89 20 ex., Gévora River, Alburquerque, Badajoz prov., Guadiana Basin, leg I. Doadrio, 8/5/87. Squalius torgalensis: MNCN243478243486, 9 ex. Mira River, Odemina, Portugal, leg I. Doadrio, 27/3/02. Squalius valentinus: holotype, Turia River, Tuejar, Valencia prov., Turia Basin, leg. P. Risueño and J. Jiménez, 13/3/03, MNCN21779598, MNCN 126706-23, 22 ex., paratypes, Algar River, Callosa D'Ensarriá, Alicante prov., Guadalest basin. Leg. CAE. 3/11/93.

Twenty-two morphometric variables were measured following Elvira (1986). All measurements are in millimetres and were log-transformed for morphometric analysis. The following abbreviations were used for morphometric and meristic characters: SL, standard length; HL, head length; PrOL, preorbital length; ED, eye diameter; AIO, Interorbital wide; PrDD, predorsal distance; PrPD, prepectoral distance; PrVD, preventral distance; PrAD, preanal distance; CPL, caudal peduncle length; APL, anal peduncle length; PVL, pectoralventral length; VAL, ventral-anal length; DFH dorsal fin heigh; DFL dorsal fin length; PFL, pectoral fin length; VFL, ventral fin length; AFL, anal fin length; $\mathrm{AFH}$, anal fin height; $\mathrm{CFL}$, caudal fin length; BD, body depth; BLD, body least depth; D, dorsal fin rays; A, anal fin rays; LLS, lateral line scales;. LTU, upper transversal scales; LTL, lower transversal scales.

The osteological characters were studied from both cleared-and-stained specimens (Wassersug, 1976). Differences in body shape among populations were analysed using Principal Component Analysis (PCA), which was performed with the statistical package NTSYS-pc (Rohlf, 1998). Burnaby's method was used to correct for size effects (Burnaby, 1966).

MoleCUlar Analysis

Fifty-two samples from seven species of Squalius from the Iberian Peninsula were analysed. Squalius alburnoides and S. palaciosi were
Table 2.- Statistical parameters for the morphometric and meristic characters of Squalius castellanus sp. nov. type series. Variables are described in Methods (SD = Standard deviation).

Tabla 2.- Parámetros estadísticos para los caracteres morfométricos y merísticos de la serie tipo de Squalius castellanus sp. nov. Las variables son descritas en los Métodos ( $\mathrm{SD}=$ desviación típica).

\begin{tabular}{lcccc}
\hline \multicolumn{5}{c}{ Squalius castellanus } \\
\cline { 3 - 5 } Variable & Holotype & Paratypes $(\mathrm{n}=20)$ \\
\hline SL & & $44.68-176.88$ & 112.98 & 47.26 \\
HL & 19.13 & $9.17-40.51$ & 25.29 & 11.24 \\
PrOL & 5.1 & $3.53-12.43$ & 8.47 & 3.27 \\
ED & 4.11 & $2.75-7.82$ & 5.81 & 1.83 \\
PrDD & 20.65 & $24.53-96.55$ & 61.67 & 25.55 \\
PrPD & 40.33 & $12.39-40.79$ & 27.95 & 10.62 \\
PrVD & 55.34 & $22.68-84.79$ & 55.63 & 22.61 \\
PrAD & 42.79 & $30.71-120.07$ & 77.82 & 31.98 \\
CPL & 26.23 & $14.82-60.4$ & 37.34 & 15.77 \\
APL & 14.94 & $9.2-35.58$ & 21.94 & 9.14 \\
PVL & 17.61 & $10.66-41.17$ & 26.90 & 11.35 \\
VAL & 13.94 & $8.55-34.28$ & 21.25 & 9.20 \\
DFL & 10.18 & $4.27-21.51$ & 13.38 & 5.561 \\
DFH & 16.72 & $8.75-29.4$ & 20.66 & 7.53 \\
PFL & 15.07 & $7.86-27.44$ & 18.64 & 7.06 \\
VFL & 14.64 & $5.46-25.79$ & 16.93 & 6.32 \\
AFL & 8.68 & $4.32-20.72$ & 11.67 & 5.11 \\
AFH & 13.67 & $7.62-24.78$ & 15.65 & 5.75 \\
CFL & 16.54 & $8.12-31.78$ & 20.48 & 7.74 \\
BD & 19.25 & $10.81-46.36$ & 28.11 & 12.05 \\
BLD & 8.89 & $5.07-19.01$ & 12.17 & 4.98 \\
AIO & 7.35 & $4.51-15.65$ & 10.74 & 4.13 \\
LLS & 40 & $39-42$ & 39.82 & 1.01 \\
LTU & 7 & $7-7$ & 7 & 0 \\
LTL & 3 & $3-3$ & 3 & 0 \\
D & 8 & $8-8$ & 8 & 0 \\
A & 7 & $7-8$ & 7.53 & 0.51 \\
\hline & & & &
\end{tabular}

not examined because these species are part of a hybridogenetic complex (Carmona et al., 1997; Alves et al., 1997, 1999, 2001; Cunha et al., 2004). Squalius lucumonis and S. keadicus from the "Mediterranean lineage" (Sanjur et al., 2003) were used as outgroup taxa. New samples examined were collected in the wild from multiple locations (Table 1). DNA was extracted from dorsal muscle, which was preserved in liquid nitrogen or $70 \%$ ethanol. Because in the last ten years only one specimen of Squalius from the Gallo River was captured (1999), we only included one specimen from this population in the molecular analysis. Posterior exhaustive samplings in this area have not reported 


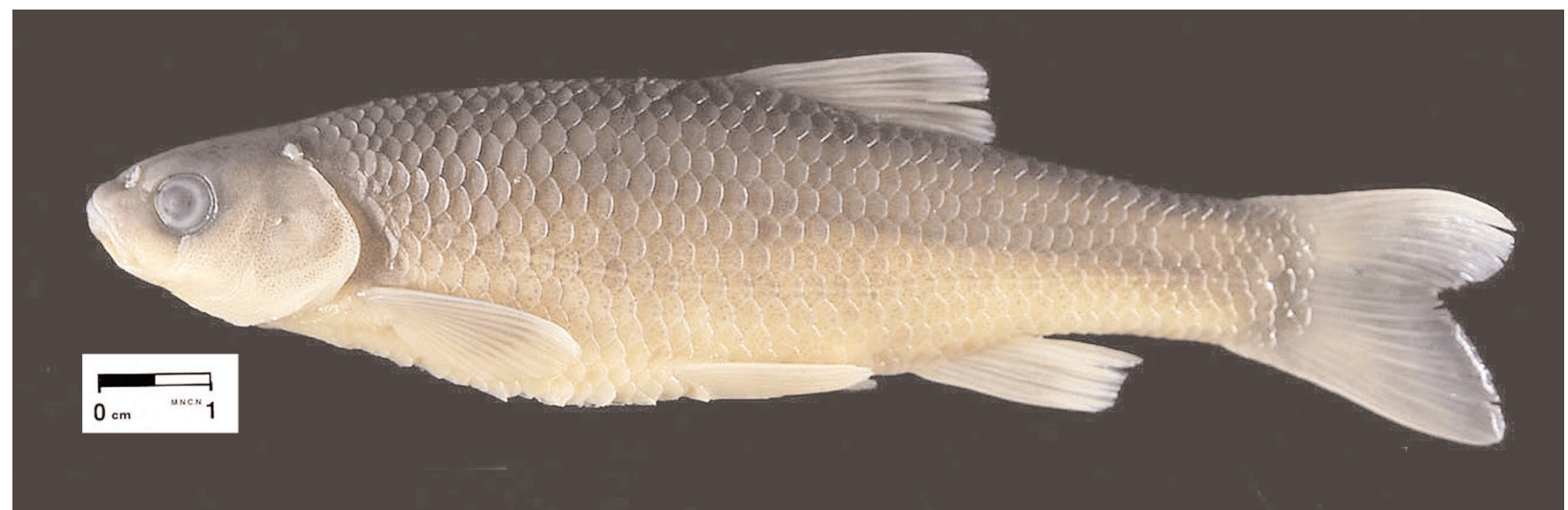

Fig. 1.- Squalius castellanus sp. nov. Holotype MNCN 212727, Gallo River, Tagus basin, Ventosa. Spain.

Fig. 1.- Squalius castellanus sp. nov. Holotype MNCN 212727, Río Gallo, Cuenca del Tajo, Ventosa. España.

new samples. Voucher specimens for this species were deposited in the collections of the Museo Nacional de Ciencias Naturales, Madrid, Spain.

Total cellular DNA was extracted from tissues by a standard proteinase $\mathrm{K}$ and phenol/chloroform extraction method (Sambrook et al., 1989) and ethanol purification (Towner, 1991). Two overlapping fragments of the mitochondrial cytochrome $b$ gene (total of $1140 \mathrm{bp}$ ) were amplified via polymerase chain reaction (PCR) from each individual DNA sample. The primers used for amplification of the cytochrome $b$ were from Machordom \& Doadrio (2001). Thermal cycle parameters were as follows: initial denaturation at $92^{\circ} \mathrm{C}(2 \mathrm{~min}), 35$ cycles of denaturation at $94^{\circ} \mathrm{C}(1$ $\mathrm{min}$ ), annealing at $48^{\circ} \mathrm{C}(1 \mathrm{~min})$, and extension at $72^{\circ} \mathrm{C}(5 \mathrm{~min})$, and a final extension at $72^{\circ} \mathrm{C}$ for 10 min. PCR mixtures were prepared in $25 \mathrm{ml}$ volumes with a final concentration of $0.5 \mathrm{ml}$ each primer, $0.2 \mathrm{mM}$ each dNTP, $1.5 \mathrm{mM} \mathrm{MgCl}$, and 1 unit of Taq DNA polymerase (Biotools). After checking PCR products on $1.5 \%$ agarose gels, they were cloned using the pGEM-T vector (Promega) into E. coli JM109, and were sequenced using the Big Dye Deoxy Ter-minator cyclesequencing kit (Applied Biosystems Inc.). DNA sequences of both strands were obtained using M13 universal (forward and reverse) sequencing primers. All samples were sequenced on an Applied Biosystems 3700 DNA sequencer following the manufacturer's instructions.

Chromatograms and alignments were visually checked and verified and there were no gaps in the resulting DNA sequences. Nucleotide saturation was analysed by plotting absolute number of tran- sitions and transversions against patristic distance values (not shown). The aligned data were analysed by Bayesian inference performed with MrBayes 3.1 (Huelsenbeck \& Ronquist, 2001) by simulating a Markov chain for 1.000 .000 cycles. Model Test vers. 3.7 (Posada \& Crandall, 1998) indicated that the best-fit model was the GTR (Rodríguez et al., 1990) + I model of evolution. Not burnin parameters were necessary.

\section{Results and discussion}

The high degree of genetic and morphological divergence of specimens from the Gallo River (Tagus Basin) to other species of Squalius of the Iberian Peninsula warrants consideration of the Gallo River population as a different species. No available name could be applied to this population, and therefore, a new species was described.

\section{Squalius castellanus sp. nov.}

Holotype: (Fig. 1, Tables 2 and 3). MNCN212727 Male, 79.13 $\mathrm{mm}$ SL. Gallo River, Ventosa, Guadalajara. leg. I. Doadrio, 30/10/99.

Paratypes: (Tables 2 and 3). MNCN136311-13, 3 ex. Arandilla River, Cuevas Labradas, Guadalajara, Gallo Basin, 13/7/44. MNCN136320-21, 2 ex. Gallo River, Molina de Aragón, Guadalajara, 13/7/44. MNCN34147-148, 2 ex. Gallo River, Cuevas Labradas, Guadalajara, leg. J. Lobón, 30/10/86. MNCN138857, 1 ex. Arandilla River, Cuevas Labradas, Guadalajara, Gallo Basin, 11/10/44. MNCN137042-45, 4 ex. Gallo River, Ventosa, Guadalajara, 13/7/44. MNCN42066-73, 8 ex. Gallo River, Cuevas Labradas, Guadalajara, leg. J. Lobón, 30/10/86. 
Table 3.- Statistical parameters for the morphometric characters of Squalius castellanus sp. nov. Each variable is divided by standard length and multiplied by ten. Variables are described in Methods ( $\mathrm{SD}=$ Standard deviation).

Tabla 3.- Parámetros estadísticos para los caracteres morfométricos de Squalius castellanus sp. nov. Cada variable está dividida por la longitud estándar y multiplicada por diez. Las variables son descritas en los Métodos ( $\mathrm{SD}=$ desviación típica).

\begin{tabular}{lcccc}
\hline \multicolumn{5}{c}{ Squalius castellanus nov. sp. } \\
\cline { 2 - 5 } Variable & Holotype & \multicolumn{3}{c}{ Paratypes $(\mathrm{n}=20)$} \\
\hline $\mathrm{SL}$ & & $44.68-176.88$ & 112.98 & 47.26 \\
$\mathrm{HL} / \mathrm{SL}$ & 79.13 & $2.1-2.3$ & 2.2 & 0.01 \\
$\mathrm{PrOL} / \mathrm{SL}$ & 2.3 & $0.6-0.8$ & 0.7 & 0.005 \\
$\mathrm{ED} / \mathrm{SL}$ & 0.7 & $0.4-0.7$ & 0.5 & 0.008 \\
$\mathrm{PrDD} / \mathrm{SL}$ & 0.5 & $2.3-2.9$ & 2.5 & 0.02 \\
$\mathrm{PrPD} / \mathrm{SL}$ & 2.6 & $4.7-5.2$ & 4.9 & 0.01 \\
$\mathrm{PrVD} / \mathrm{SL}$ & 5.1 & $6.7-7.2$ & 6.9 & 0.02 \\
$\mathrm{PrAD} / \mathrm{SL}$ & 7.0 & $5.2-5.6$ & 5.5 & 0.01 \\
$\mathrm{CPL} / \mathrm{SL}$ & 5.4 & $3.2-3.4$ & 3.2 & 0.007 \\
$\mathrm{APL} / \mathrm{SL}$ & 3.3 & $1.8-2.1$ & 1.9 & 0.009 \\
$\mathrm{PVL} / \mathrm{SL}$ & 1.9 & $2.2-2.5$ & 2.4 & 0.007 \\
$\mathrm{VAL} / \mathrm{SL}$ & 2.2 & $1.7-2.0$ & 1.9 & 0.009 \\
$\mathrm{DFL} / \mathrm{SL}$ & 1.8 & $0.9-1.3$ & 1.2 & 0.01 \\
$\mathrm{DFH} / \mathrm{SL}$ & 1.9 & $1.6-2.2$ & 1.9 & 0.02 \\
$\mathrm{PFL} / \mathrm{SL}$ & 2.1 & $1.4-1.9$ & 1.7 & 0.01 \\
$\mathrm{VFL} / \mathrm{SL}$ & 1.9 & $1.2-1.8$ & 1.5 & 0.01 \\
$\mathrm{AFL} / \mathrm{SL}$ & 1.8 & $0.8-1.2$ & 1 & 0.01 \\
$\mathrm{AFH} / \mathrm{SL}$ & 1.1 & $1.3-1.9$ & 1.5 & 0.02 \\
$\mathrm{CFL} / \mathrm{SL}$ & 1.7 & $1.7-2.1$ & 1.8 & 0.01 \\
$\mathrm{BD} / \mathrm{SL}$ & 2.1 & $2.3-2.6$ & 2.5 & 0.009 \\
$\mathrm{BLD} / \mathrm{SL}$ & 2.4 & $1-1.1$ & 1.1 & 0.003 \\
$\mathrm{AIO} / \mathrm{SL}$ & 1.1 & $0.9-1.1$ & 1 & 0.007 \\
\hline
\end{tabular}

DiAGNOSIS: Differs from all other known species of Squalius by the following combination of characters: Seven to eight branched rays on the anal fin; A short and wide head, 4.3-4.9 (mean = 4.5) times the standard length; Short pectoral-ventral length, reaching 2.2-2.4 (mean $=2.3)$ times the stan- dard length; Number of scales on lateral line mean $=39.8$ (39-42); Number of scales on upper transversal line mean $=7$. Three scales on lower transversal line. Large infraorbitals bones that do not cover the adductor mandibulae muscle. Twenty autapomorphies in the mitochondrial cytochrome $b$ gene.

DESCRIPTION: D II-III 8, A II-III 7-8 $($ mean $=7.5)$, P I 13, V II 7, C 18, LLS 39-42 $($ mean = 39.8), A medium sized species that rarely reaches a standard length of $200 \mathrm{~mm}$. Morphometric characters are given in Tables 2 and 3. Maximum body depth is 3.8-4.2 (mean $=4)$ times the standard length. Head short and is 4.3-4.9 (mean $=4.5$ ) times the standard length. Head length is lesser than maximum body depth. Preorbital distance is longer than eye diameter, reaching 1.2-1.6 $($ mean $=1.4)$ times the eye diameter. There is a long interorbital distance, reaching 1.6-2 $($ mean $=1.8)$ times the eye diameter. Ventral fin is inserted slightly before origin of dorsal fin. Predorsal length 1.1-1.2 (mean $=1.1$ ) times preventral length. High caudal peduncle. The ventral-anal distance is long, with an anal index (VAL/PRAD) of 2.4-2.9 (mean = 2.7). Minimum body depth is 2.9$3.2($ mean $=3)$ times length of caudal peduncle and 1.7-1.9 (mean $=1.8)$ times length of anal peduncle. The pectoral-ventral distance is short, reaching 2.22.4 (mean $=2.3$ ). Fin size is moderately large.

PigmeNTATION PATTERN: Silver body, darker dorsally. There is a black spot at the base of the scales that appears to be divided by the lateral line pore into two separate spots. The peritoneum is silver with small black spots.

Distribution: This new species is endemic to the Gallo River and its tributaries in the upper Tagus Basin (Central Spain) (Fig. 2).

Etymology: The species name "castellanus" derives from the name of the Spanish region -Castille- inhabited by this species. An adjective.

Table 4.- Autapomorphies in cytochrome $b$ gene in Squalius castellanus. Transversions are indicated with *.

Tabla 4.- Autopomorfías en el gen citocromo b en Squalius castellanus. Las transversiones se indican con *.

\begin{tabular}{lcccccccccccccccccccc}
\hline Cytochrome b position & $\mathbf{1 0 1}$ & $\mathbf{1 7 9}$ & $\mathbf{3 0 2}$ & $\mathbf{3 3 2}$ & $\mathbf{4 3 4}$ & $\mathbf{4 6 4}$ & $\mathbf{4 9 4}$ & $\mathbf{4 9 7}$ & $\mathbf{5 0 3}$ & $\mathbf{6 1 6}$ & $\mathbf{7 2 2}$ & $\mathbf{7 4 7}$ & $\mathbf{7 5 5}$ & $\mathbf{8 6 1}$ & $\mathbf{8 7 8}$ & $\mathbf{8 9 6}$ & $\mathbf{9 0 5}$ & $\mathbf{9 3 8}$ & $\mathbf{1 0 8 8}$ & $\mathbf{1 1 0 0}$ \\
\hline Squalius castellanus & $\mathrm{C}$ & $\mathrm{T}$ & $\mathrm{C}$ & $\mathrm{G}$ & $\mathrm{G}$ & $\mathrm{T}$ & $\mathrm{A}$ & $\mathrm{G}$ & $\mathrm{T}$ & $\mathrm{C}$ & $\mathrm{C}$ & $\mathrm{T}$ & $\mathrm{T}$ & $\mathrm{T}$ & $\mathrm{G}$ & $\mathrm{A}$ & $\mathrm{G}$ & $\mathrm{G}$ & $\mathrm{A}$ & $\mathrm{G}$ \\
Squalius carolitertii & $\mathrm{G}$ & $\mathrm{G}$ & $\mathrm{T}$ & $\mathrm{A}$ & $\mathrm{A}$ & $\mathrm{C}$ & $\mathrm{G}$ & $\mathrm{T}$ & $\mathrm{C}$ & $\mathrm{A}$ & $\mathrm{T}$ & $\mathrm{C}$ & $\mathrm{C}$ & $\mathrm{C}$ & $\mathrm{A}$ & $\mathrm{G}$ & $\mathrm{A}$ & $\mathrm{A}$ & $\mathrm{C}$ & $\mathrm{A}$ \\
Squalius pyrenaicus & $\mathrm{A}$ & $\mathrm{A}$ & $\mathrm{T}$ & $\mathrm{A}$ & $\mathrm{A}$ & $\mathrm{C}$ & $\mathrm{G}$ & $\mathrm{A}$ & $\mathrm{C}$ & $\mathrm{A}$ & $\mathrm{T}$ & $\mathrm{C}$ & $\mathrm{C}$ & $\mathrm{C}$ & $\mathrm{A}$ & $\mathrm{G}$ & $\mathrm{A}$ & $\mathrm{A}$ & $\mathrm{C}$ & $\mathrm{A}$ \\
\hline
\end{tabular}




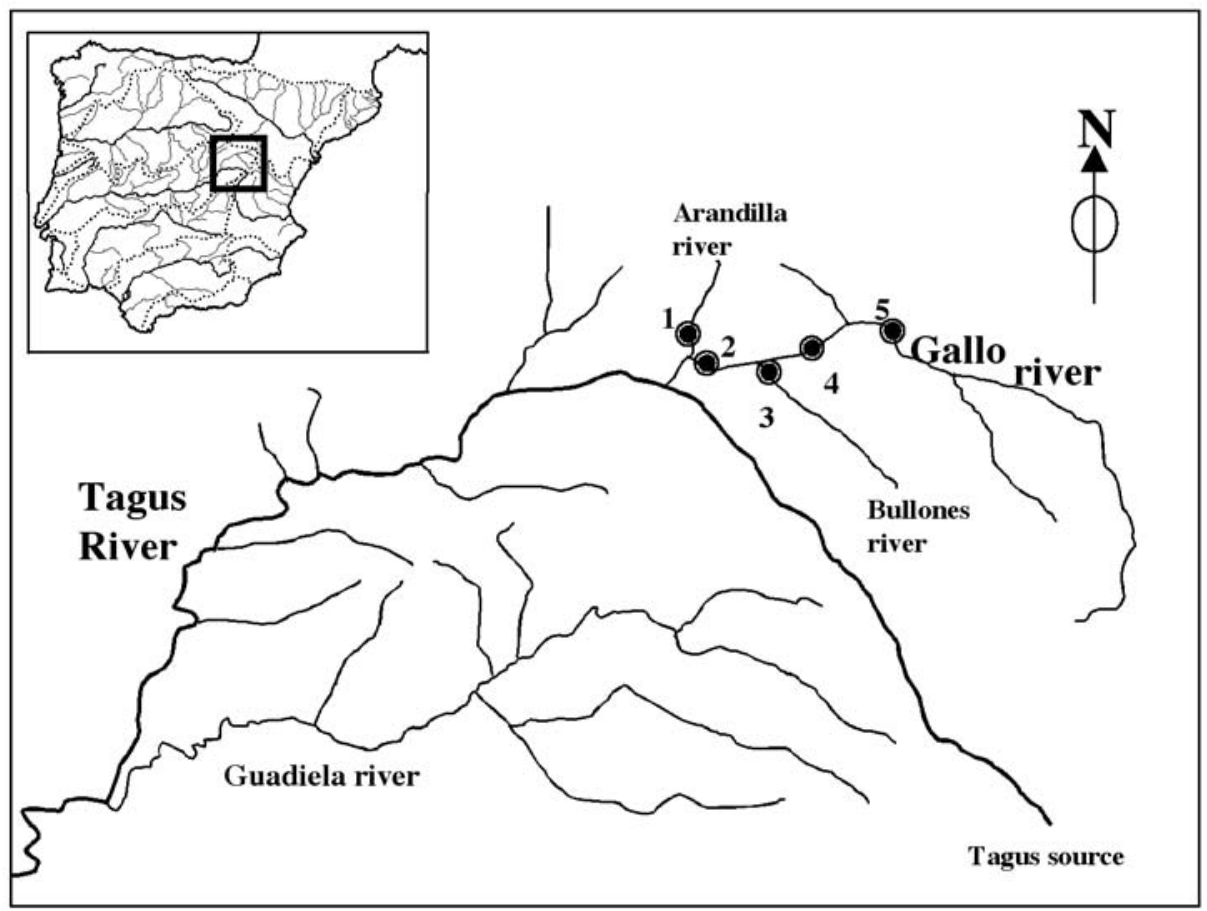

Fig. 2.- Locations sites of Squalius castellanus. 1. Arandilla river (Cuevas Labradas); 2. Gallo river (Cuevas Labradas); 3. Bullones river (Torete); 4. Gallo river (Ventosa); 5. Gallo river (Molina de Aragón).

Fig. 2.- Localidades de Squalius castellanus. 1. Río Arandilla (Cuevas Labradas); 2. Río Gallo (Cuevas Labradas): 3. Río Bullones (Torete); 4. Río Gallo (Ventosa); 5. Río Gallo (Molina de Aragón).

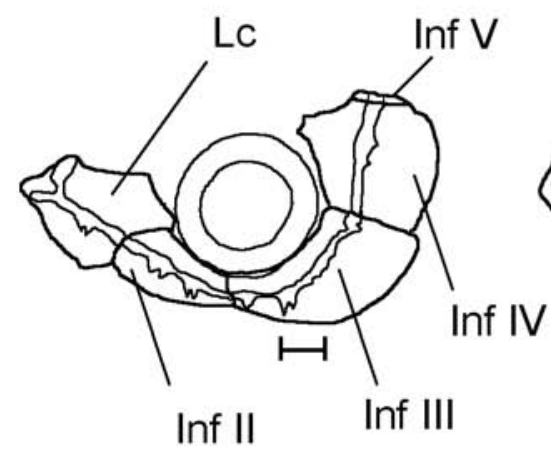

A

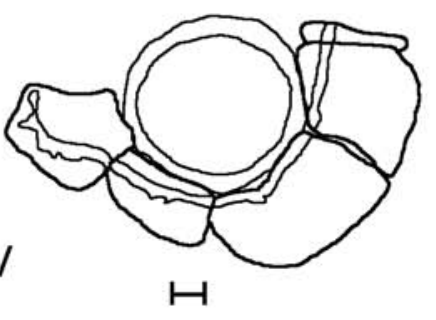

B

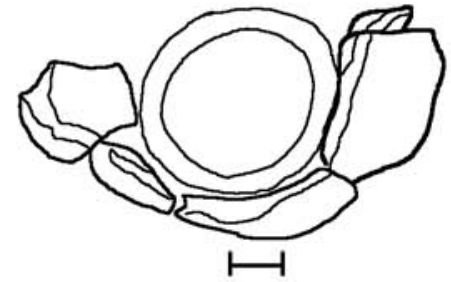

C

Fig. 3.- Infraorbital bones of Iberian Squalius species. Lc: Lacrimal bone; Inf II: Second infraorbital bone; Inf III: Third infraorbital bone; Inf IV: Fourth infraorbital bone; Inf V: Fifth infraorbital bone. A: Squalius castellanus; B: Squalius pyrenaicus; C: Squalius carolitertii.

Fig. 3.- Huesos infraorbitarios de las especies ibéricas de Squalius. Lc: hueso lacrimal; Inf II: Segundo hueso infraorbitario; Inf III: Tercer hueso infraorbitario; Inf IV: Cuarto hueso infraorbitario; Inf V: Quinto hueso infraorbitario. A: Squalius castellanus; B: Squalius pyrenaicus; C: Squalius carolitertii. 
Table 5.- Summary of diagnostic morphological and meristic characters of Squalius castellanus and differences with S. carolitertii and $S$. pyrenaicus.

Tabla 5.- Resumen de los caracteres morfológicos y merísticos diagnósticos de Squalius castellanus y diferencias con $S$. carolitertii y $S$. pyrenaicus.

\begin{tabular}{|c|c|c|c|}
\hline$\overline{\text { Characters }}$ & S. castellanus sp nov & S. carolitertii & S. pyrenaicus \\
\hline Number of scales in lateral line & $39-42($ mean $=39.8)$ & $40-43($ mean $=41.6)$ & $38-44($ mean $=41.3)$ \\
\hline Number of scales in upper transversal line & 7 & 8 & $8-9$ \\
\hline Head & $\begin{array}{l}\text { Short and wide } \\
\text { Cephalic index } \\
(\mathrm{HL} / \mathrm{SL})=2.0-2.3\end{array}$ & $\begin{array}{l}\text { Long and narrow } \\
\text { Cephalic index } \\
(\mathrm{HL} / \mathrm{SL})=2.3-2.5\end{array}$ & $\begin{array}{l}\text { Long and narrow } \\
\text { Cephalic index } \\
(\mathrm{HL} / \mathrm{SL})=2.6-2.8\end{array}$ \\
\hline Infraorbital bone & $\begin{array}{c}\text { Wide } \\
\text { Cover parcially the } \\
\text { muscle adductor mandibulae }\end{array}$ & Narrow & $\begin{array}{l}\text { Wide } \\
\text { Cover totally the muscle } \\
\text { adductor mandibulae }\end{array}$ \\
\hline Pectoral-Ventral length & $\begin{array}{c}\text { Short } \\
\text { Ventral index } \\
(\mathrm{PVL} / \mathrm{PRDD})=4.1-4.5\end{array}$ & $\begin{array}{c}\text { Long } \\
\text { Ventral index } \\
(\mathrm{PVL} / \mathrm{PRDD})=4.6-5\end{array}$ & $\begin{array}{c}\text { Long } \\
\text { Ventral index } \\
(\mathrm{PVL} / \mathrm{PRDD})=3.3-4.9\end{array}$ \\
\hline Ventral-Anal length & $\begin{array}{c}\text { Short } \\
\text { Ventral-Anal index } \\
(\mathrm{VAL} / \mathrm{SL})=1.7-1.9\end{array}$ & $\begin{array}{c}\text { Long } \\
\text { Ventral-Anal index } \\
(\mathrm{VAL} / \mathrm{SL})=1.8-2.1\end{array}$ & $\begin{array}{c}\text { Short } \\
\text { Ventral-Anal index } \\
(\mathrm{VAL} / \mathrm{SL})=1.2-2.0\end{array}$ \\
\hline
\end{tabular}

COMMON NAMES: We propose using the common name "bordallo del Gallo" in Spanish, and "Gallo chub" in English.

CONSERvation: Squalius castellanus is only known from small populations along the Gallo River (Tagus basin) and its main tributaries, both Bullones and Arandilla Rivers. In the last ten years only one individual has been collected in the Gallo River. The main threat to the species is pollution from the city of Molina de Aragon. Consequently, the population is currently declining and should be considered Critically Endangered (CR B1 + 2ce) according to the IUCN Red List Categories.

GENETICS: The genetic distance to the other Iberian species was the following: $3.7-4.2$ (mean $_{\mathrm{GTR}}=3.8$ ) to S. carolitertii, 5.7-6.6 $\left(\right.$ mean $\left._{\mathrm{GTR}}=6.1\right)$ to $S$. pyrenaicus, 6.6 to $S$. valentinus, 9.2 to $S$. malacitanus, 11.7-11.9 $\left(\right.$ mean $\left._{\mathrm{GTR}}=11.8\right)$ to $S$. aradensis and 12.9 to $S$. torgalensis. Squalius castellanus has twenty autapomorphies in the cytochrome $b$ gene, five of which are transversions (Table 4).

INTERESPECIFIC COMPARISONS: Specimens of Squalius from the Gallo River (Tagus basin) show many differences in both genetic and morphological characters with respect to $S$. carolitertii and $S$. pyrenaicus. Thus, this population deserves recognition as a new species, Squalius castellanus. On Table 5 there is a summary of the main differences between the new species $S$. castellanus and both $S$.
Table 6.- Eigenvectors for the first three principal components Burnaby transformed for 21 variables.

Tabla 6.- Eigenvectors para los tres primeros ejes principales de las 21 variables transformadas mediante Burnaby.

\begin{tabular}{lccc}
\hline & C1 & C2 & C3 \\
\hline SL & 0.0049 & 0.0041 & 0.0041 \\
HL & 0.0295 & 0.0113 & 0.0131 \\
PRO & -0.0206 & 0.0034 & 0.0094 \\
ED & 0.0259 & 0.0064 & 0.0041 \\
PrDD & 0.0033 & 0.0004 & -0.0034 \\
PrPD & 0.0044 & 0.0030 & 0.0047 \\
PrPV & 0.0018 & 0.0019 & -0.0015 \\
PrAD & 0.0009 & 0.0036 & 0.0005 \\
CPL & 0.0278 & 0.0047 & -0.0007 \\
APL & 0.0294 & 0.0129 & -0.0007 \\
PVL & -0.0212 & -0.0002 & -0.0087 \\
VAL & -0.0404 & 0.0313 & -0.0039 \\
DFL & -0.0059 & -0.0158 & 0.0207 \\
DHL & -0.0140 & -0.0167 & 0.0115 \\
PFL & 0.0041 & -0.0154 & -0.0152 \\
VFL & 0.0046 & -0.0126 & -0.0039 \\
AFL & -0.0064 & -0.0133 & -0.0165 \\
AHL & -0.0027 & -0.0191 & -0.0016 \\
BD & 0.0079 & -0.0026 & -0.0057 \\
BLD & -0.0066 & 0.0009 & -0.0005 \\
AIO & -0.0105 & 0.0034 & 0.0005 \\
Eigenvalues & 0.113 & 0.018 & 0.001 \\
& $(79.5 \%)$ & $(13.1 \%)$ & $(1.1 \%)$ \\
\hline & & &
\end{tabular}




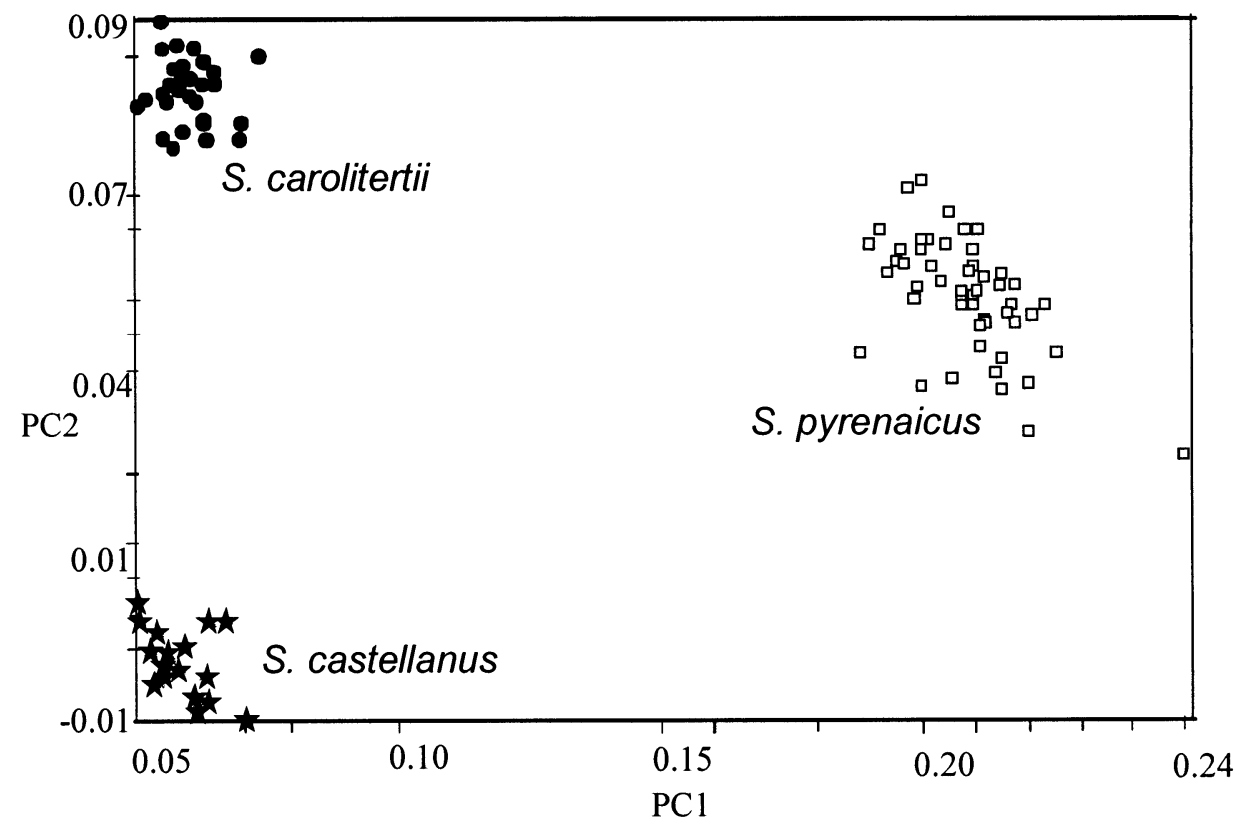

Fig. 4.- Bivariate plot of Principal Component 2 vs. Principal Componet 1 for 21 morphometric Burnaby corrected variables. $S$. pyrenaicus $(\mathrm{n}=53) ;$ S. carolitertii $(\mathrm{n}=30) ;$ S. castellanus $(\mathrm{n}=17)$.

Fig. 4.- Representación bivariante del componente principal 2 vs. el componente principal 1 para 21 variables morfométricas corregidas por el método Burnaby. S. pyrenaicus $(\mathrm{n}=53)$; S. carolitertii $(\mathrm{n}=30) ;$. castellanus $(\mathrm{n}=17)$.

carolitertii and S. pyrenaicus. Squalius castellanus differs from $S$. carolitertii and $S$. pyrenaicus by having a shorter head (2.0-2.3 HL/SL vs. 2.3-2.5 in S. carolitertii and $2.6-2.8$ in $S$. pyrenaicus), and a shorter pectoral-ventral distance (4.1-4.5 PVL/PRDD vs. 4.6-5 in $S$. carolitertii and 3.3-4.9 in $S$. pyrenaicus). The ventral-anal distance is longer than $S$. pyrenaicus, with an anal index $\mathrm{VAL} / \mathrm{SL}=1.7-1.9$ in $S$. castellanus vs. 1.2-2.0 in $S$. pyrenaicus, and is shorter than $S$. carolitertii (anal index VAL/SL: 1.7-1.9 in S. castellanus vs. 1.8-2.1 in $S$. carolitertii). The number of scales in the upper transversal row is 7 in Squalius castellanus vs. 8 scales in $S$. carolitertii and mean $=8(8-9)$ in $S$. pyrenaicus. The infraorbital bones of $S$. castellanus differ from the other Iberian Squalius species by both the shape and the position of the adductormandibulae muscle. The infraorbital bones are wider in $S$. castellanus than in $S$. carolitertii (Fig. 3 ). In adult specimens of $S$. pyrenaicus, the adductor-mandibulae muscle is practically covered by the infraorbital bones, while in S. castellanus the bones only cover half of muscle. The PCA analysis with 21 morphometric variables using Burnaby's (1966) size correction found significant differences in morphology between $S$. carolitertii, $S$. castellanus and S. pyrenaicus (Fig. 4). The highest eigenvector for both, the one and second component was VAL (Table 6). The first three components accumulate $93.66 \%$ of variance (Table 6).

Phylogenetic Relationships: Phylogenetic analysis of mt-cytochrome $b$ sequences using Bayesian analyses (Huelsenbeck \& Ronquist 2001) found that the Iberian species belonging to the "Mediterranean lineage" did not reject the monophyletic hypothesis to this lineage proposed by Sanjur et al. (2003). Three main clades with high posterior probabilities support were recognized in this lineage: $S$. aradensis plus $S$. torgalensis; $S$. pyrenaicus populations together with $S$. malacitanus and $S$. valentinus; and a third clade consisting of $S$. carolitertii and $S$. castellanus. (see Fig. 5 ). These clades have been recognized by other authors (Brito et al., 1997; Sanjur et al., 2003; Doadrio \& Carmona, 2006), but the position of $S$. castellanus was never resolved. The genetic divergence of $S$. castellanus was smaller in relation to $S$. 


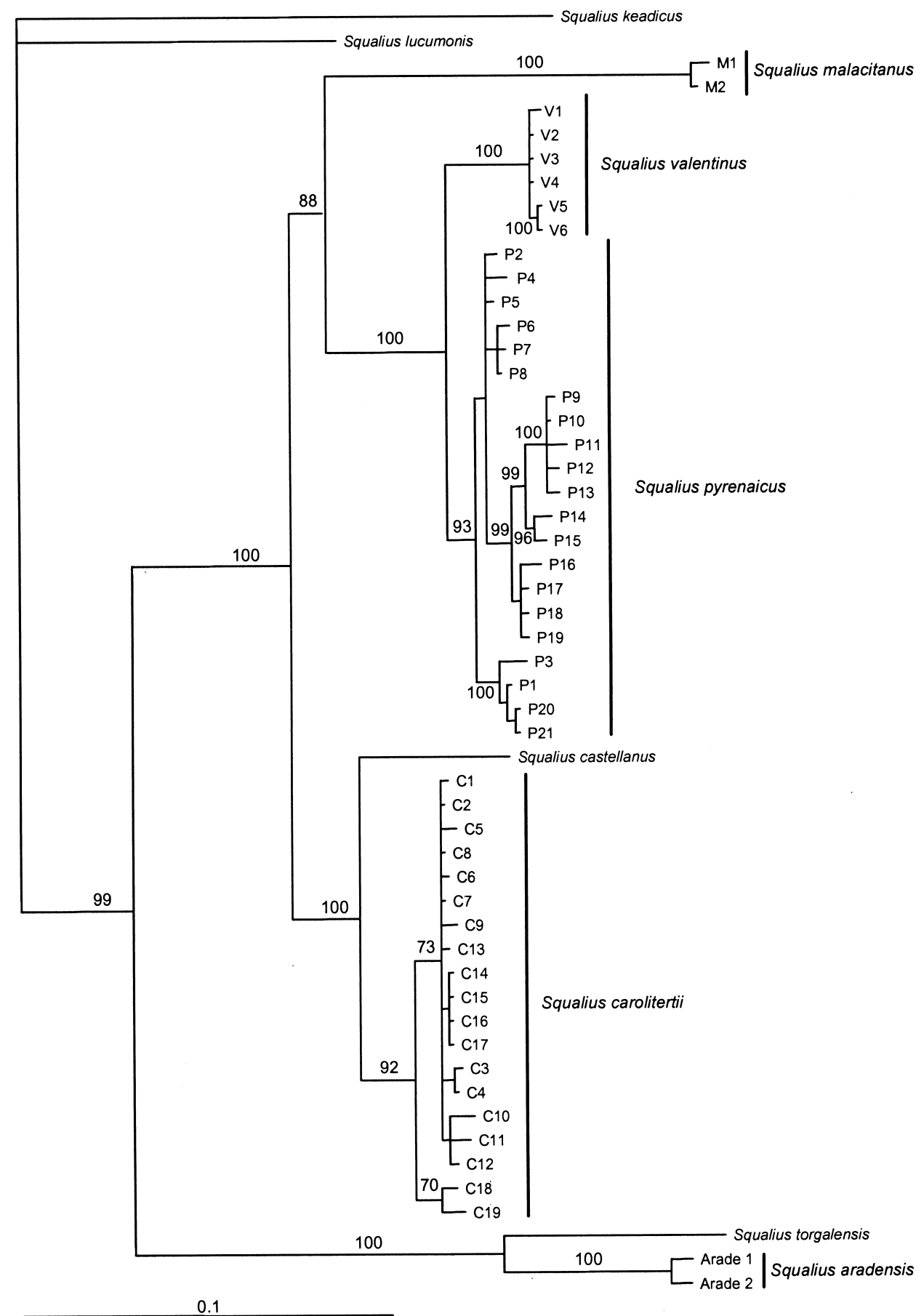

Fig. 5.- Phylogenetic tree of 52 analysed specimens of genus Squalius recovered from cytochrome $b$ sequences according to the bayesian method. Branch lengths are proportional to the estimated mean number of substitutions per site. Numbers are posterior probabilities of bayesian analysis.

Fig. 5.- Árbol filogenético de 52 especímenes del género Squalius obtenido a partir de secuencias de citocromo $b$ analizadas mediante inferencia Bayesiana. Las longitudes de las ramas son proporcionales al número medio de sustituciones por sitio. Los números indican las probabilidades posteriores del análisis bayesiano. 
carolitertii $\left(\operatorname{mean}_{\mathrm{GTR}}=3.8,3.7-4.2\right)$ than to $S$. pyrenaicus $\left(\operatorname{mean}_{\mathrm{GTR}}=6.1,5.7-6.6\right)$. In respect of $S$. aradensis and $S$. torgalensis, the genetic distances found between these species and $S$. castellanus were the highest $\left(\right.$ mean $_{\mathrm{GTR}}=11.8,11.7-11.9$ and mean $_{\text {GTR }}=12.9$ respectively). If a molecular clock with a $1 \%$ rate of mutation per $1 \mathrm{My}$ is assumed for the mt cytochrome $b$ gene (Dowling et al., 2002; Doadrio \& Carmona, 2004), the Iberian Squalius species belonging to the "Mediterranean lineage" diverged from other European species in the upper Miocene period (13 MYA). This divergence was possible when Asia Minor, Greece, the Balkans, Italy and the Iberian Peninsula were geographically connected. The break up of this geographical connection may have provided the conditions for the origin of Iberian Squalius species that subsequently became isolated populations that experienced further allopatric speciation (Sanjur et al., 2003). The complexity of the Iberian hydrographic basins in the Tertiary period contributed to allopatric speciation within the Iberian Peninsula, and led to the current diversity of Iberian endemic species. According to the molecular clock for cytochrome $b$ (Dowling et al., 2002; Doadrio \& Carmona, 2004), the separation between the new species described, S. castellanus, and the species genetically closer to it, $S$. carolitertii, is coincident with the lower Pliocene (3.8 MYA). Not geological events during the Pliocene could be claimed to explain the colonization and posterior speciation of S. castellanus.

\section{AKNOWLEDGEMENTS}

We would like to thank the Regional Administration of Castilla-La Mancha for permission to collect the Squalius samples. We gratefully acknowledge the field help of L. Alcaraz, F. Alda, P. Cabezas, F. Morcillo, L. Kalous and P. Ornelas. L. Alcaraz also assisted in laboratory work. R. Pérez Rodríguez collaborates with us in statistical analysis. This research was supported by projects REM 2001-0662/G10 and 07M/0109/02. Sarah Young further revised the English text.

\section{References}

Alves, M. J., Coelho, M. M. \& Collares-Pereira, M. J., 1997. The Rutilus alburnoides complex (Cyprinidae): evidence for hybrid origin. Journal of Zoological Systematics and Evolutionary Research, 35:1-10.

Alves, M. J., Coelho, M. M. \& Collares-Pereira, M. J., 2001. Evolution in action through hybridization and polyploidy in an Iberian fresh water fish: a genetic review. Genetica, 111(1-3): 375-385.
Alves, M. J., Coelho, M. M., Prospero, M. I. \& Collares-Pereira, M. J., 1999. Production of fertile unreduced sperm by hybrids males of the Rutilus alburnoides complex (Teleostei, Cyprinidae): an alternative route to genome tetraploidization in unisexuals. Genetics, 151: 277-283.

Brito, R. M., Briolay, J., Galtier, N., Bouvet, Y. \& Coelho, M. M., 1997. Phylogenetic relationships within genus Leuciscus (Pisces: Cyprinidae) in Portuguese freshwaters, based on mitochondrial DNA cythocrome b sequences. Molecular Phylogenetics and Evolution, 8: 435-442.

Burnaby, T. P., 1966. Growth-invariant discriminant functions and generalized distances. Biometrics, 22: 96-110.

Carmona, J. A., SAnjur, O. I., Doadrio, I., Machordom, A. \& VRIJENHOEK, R. C., 1997. Hybridogenetic reproduction and maternal ancestry of polyploid Iberian fish: the Tropidophoxinellus alburnoides complex. Genetics, 146: 983-993.

Coelho, M. M., Brito, R. M., Pacheco, T. R., Figueiredo, D. \& PIRES, A. M., 1995. Genetic variation and divergence of Leuciscus pyrenaicus and Leuciscus carolitertii (Pisces, Cyprinidae). Journal of Fish Biology, 47: 243-258.

Cunha, C., Coelho, M. M., Carmona, J. A. \& Doadrio, I., 2004. Phylogeographical insights into the origins of the Squalius alburnoides complex via multiple hybridization events. Molecular Ecology, 13: 2807-2817.

DoAdrio, I. \& CARMONA, J. A., 1998. Genetic divergence in Greek populations of the genus Leuciscus and its evolutionary and biogeographical implications. Journal of Fish Biology, 53: 591-613.

DoAdrio, I. \& CARMONA, J. A., 2003. Testing freshwater Lago Mare dispersal theory on the phylogeny relationships of Iberian cyprinid genera, Chondrostoma and Squalius. Graellsia, 59(2-3): 457-473.

DoAdrio, I. \& CARMONA, J. A., 2004. Phylogenetic relationships and biogeography of the genus Chondrostoma inferred from mitochondrial DNA sequences. Molecular Phylogenetics and Evolution, 33: 802-815.

DoAdrio, I. \& CARmona, J. A., 2006. Phylogenetic overview of the genus Squalius (Actinopterygii, Cyprinidae) in the Iberian Peninsula, with description of two new species. Cybium, 30(3): 199-214.

Doadrio, I., Carmona, J. A. \& Fernández-Delgado, C., 2002. Morphometric study of the Iberian Aphanius (Actinopterygii, Cyprinodontiformes), with description of a new species. Folia Zoologica, 51(1): 67-79.

Dowling, T. E., TibBets, C. A., Minckley, W. L. \& Sмith, G. R., 2002. Evolutionary relationships of the plagopterins (Teleostei: Cyprinidae) from Cytochrome b sequences. Copeia, 2002(3): 655-678.

Durand, J. D., Unlü, E., DoAdrio, I., Pipoyan, S. \& Templeton, A. R., 2000. Origin, radiation, dispersion and allopatric hybridization in the chub Leuciscus 
cephalus. Proceedings of the Royal Society of London, B267: 1687-1697.

ElviRA, B., 1986. Revisión taxonómica y distribución geográfica del género Chondrostoma Agassiz, 1835 (Pises, Cyprinidae). $\mathrm{PhD}$. Thesis. Instituto Nacional de Investigaciones Agrarias. Madrid. 530 pp.

Huelsenbeck, J. P. \& Ronquist, F., 2001. Mr Bayes: Bayesian inference of phylogenetic trees. Bioinformatics, 17(8): 754-755.

Kottelat, M., 1997. European freshwater fishes. Biologia, 52(Suppl. 5): 1-271.

MACHORDOM, A. \& DoAdRIO, I., 2001. Evidence of a Cenozoic Betic-Kabilian connection based on a freshwater fish phylogeography (Luciobarbus, Cyprinidae). Molecular Phylogenetics and Evolution, 18: 252-263.

Posada, D. \& CRANDAll, K.A., 1998. MODELTEST: testing the model od DNA substitution. Bioinformatics, 14(9): 817-818.

Rodríguez, F., Oliver, J. F., Marín, A. \& Medina, J. R., 1990. The general stochastic model of nucleotide substitutions. Journal of Theorical Biology, 142: 485-501.

ROHLF, F., 1998. NTSYS-pc numerical taxonomy and multivariate analysis system. Version 2.02. Exeter Publications Setauket, New York.

Sambrook, J., Fritsch, E. F. \& Maniatis, T., 1989. Molecular cloning: A Laboratory Manual. Second ed. Cold Spring Harbor Laboratory Press. Cold Spring Harbor.
Sanjur, O. I., Carmona, J. A. \& Doadrio, I., 2003. Evolutionay and biogeographical patterns within Iberian populations of the genus Squalius inferred from molecular data. Molecular Phylogenetics and Evolution, 29: 20-30.

TOWNER, P., 1991. Purification of DNA. In: Brown, T. A. (Ed). Essential Molecular Biology. A practical approach. Oxford University Press. Oxford: 47-68.

WASSERsug, R. J., 1976. A procedure for differential staining of cartilage and bone in whole formalinfixed vertebrates. Stain Technology, 51: 131-134.

ZARDOYA, R. \& DOADRIO, I., 1999. Molecular evidence on the evolutionary and biogeographical patterns of European Cyprinids: Systematic and biogeographical implications. Proceedings of the Royal Society of London, B265: 1365-1372.
Recibido, 7-II-2007

Aceptado, 4-V-2007 Publicado, 27-VI-2007 